

Published in final edited form as:

Conf Proc IEEE Eng Med Biol Soc. 2011 ; 2011: 7462-7465. doi:10.1109/IEMBS.2011.6091750.

\title{
Design And Evaluation Of a Prosthetic Shoulder Controller
}

\author{
Joseph E. Barton [Member IEEE] \\ Neurology and the Physical Therapy \& Rehabilitation Science Departments, University of \\ Maryland School of Medicine, Baltimore, MD. 21201 USA, and the Veterans Administration \\ Rehabilitation Research \& Development Service, Baltimore MD 21201, USA (410-706-5211; fax: \\ 410-706-4903;)
}

Joseph E. Barton: jbarton@som.umaryland.edu

\begin{abstract}
The first goal of this study was to develop a 2 degree of freedom (DOF) upper limb controller utilizing shoulder elevation/depression and protraction/retraction. Its primary purpose is to control powered prosthetic shoulder joints being incorporated into a new generation of prosthetic arms for shoulder disarticulation and very high transhumeral amputees. More generally, however, such a controller can be applied to simultaneously control any 2 DOF's under a direct, proportional control scheme. There is no generally accepted method to objectively and quantitatively evaluate prosthesis and prosthesis control performance, and the second goal of this study was to develop one based on Fitts Law. The evaluation protocol has quite general applicability as well, and can be used to compare different reaching/pointing devices, or to compare variations in the design or operation of a particular device.
\end{abstract}

\section{Introduction}

The control of upper limb prostheses via shoulder movement has been employed since the civil war. Historically, amputees controlled such devices with movements of their trunk and intact limbs and joints. An amputee would, for example, pull on a cable with a shoulder harness by protracting his or her shoulders, positioning one component of the prosthesis. When this component was positioned, the amputee would hit a switch or a touch sensor with his/her chin or other hand to lock that component in place and then operate the next component. Cable and harness systems such as these are bulky and cumbersome, and are incapable of providing fast, fluid, and precise control of prosthesis. Bayer and colleagues [1, 2] developed a $2 \mathrm{DOF}$ shoulder position transducer for use by quadriplegic patients in controlling powered wheel chairs. The transducer consisted of a load cell fixed over the patient's sternum with double-sided tape. A length of piano wire extended from the load cell to the front of the shoulder, where it too was fixed with double sided tape. All of the necessary electronics were packaged with the load cell, which sensed directional tension of the piano wire resulting from shoulder elevation/depression and protraction/retraction. After several hours of training, patients were able to hold and move to 10 to 20 distinct points along each axis of the shoulder; and also achieved impressive performance in operating their powered wheel chairs.

The first goal of this development was to build upon Bayer et al's approach and develop a feedback controller, where the feedback was a resistive force proportional to shoulder 
displacement. This in turn required quantification of the shoulder's range of motion, as well as the forces it is capable of exerting in each direction (more specifically, motion and forces at the point of the acromion process.) Beginning with the classic works of Inman, et al, a wealth of anatomical $[3,4]$ and anthropometric $[5,6]$ investigations of the shoulder complex have been undertaken in modern times. More recently, several biomechanical models have also been developed [7, 8]. Very little if any data exists, however, quantifying the movements and forces exerted at the acromion process. These measurements were therefore also undertaken as part of this study.

Currently there is no generally accepted method to objectively and quantitatively evaluate prosthesis and prosthesis control performance, and the second goal of this study was to develop such a method. Perhaps the most important function of the human upper limb is to grasp and/or manipulate objects with the hand, but before this can be done it must be quickly and accurately placed at a specific location in space by the arm and shoulder. The arm and shoulder can thus be thought of as a pointing device, for which there is a widely accepted evaluation method based on the work of Fitts [9], who introduced a quantitative measure of the difficulty in moving from a starting point to a particular target. Fitts' method is used extensively in the evaluation of computer pointing devices [10]. A computer-based implementation of the method was developed for this study in which targets are arranged on a display monitor and test subjects move a cursor from one to the next with shoulder movements sensed by the candidate shoulder controllers.

\section{METHODS}

\section{A. Goal 1}

Four candidate control assemblies were assessed. The first three consisted of a one-piece, machined aluminum base which fixed to a body socket at the base of the neck (Fig. 1). Steel rods of varying diameter (and thus stiffness) (Table 1) were then press fit into a hole drilled into the cantilever end of the base. The steel rods imparted a resistive force proportional to shoulder displacement. Subjectively, rod stiffness ranged from just-noticeable to very noticeable. Four strain gauges were mounted on each face of the square aluminum cantilever end and arranged in a Wheatstone Bridge to sense bending of the rod. The bridge's output was utilized as the control signal. The fourth assembly, a 2 DOF potentiometer, offered no resistance to motion. We tested these assemblies in a position control scheme (controlled e element position is proportional to shoulder position) and a velocity control scheme (direction and velocity are proportional).

Shoulder movement was measured using the apparatus shown in Fig. 2. It consisted of a body socket on an adjustable stand, which was positioned over the test subject's left shoulder. Three steel tubes were inserted through the socket at or across from the acromion process. With the test subject sitting in the shoulder-neutral position, a steel rod was passed through the tube and placed in contact with the subject's shoulder. This initial position was then marked. The subject was then instructed to move his or her shoulder towards or away from the rod to a comfortably maximal position. (For the controller to be practicable, both the range of motion and the range of force production should be constrained to that which can be comfortably achieved by the wearer. Though this may change from individual to 
individual and from socket to socket, the goal here was to specify approximately what these ranges would be.) A second mark was placed on the rod and shoulder movement in the desired direction recorded as the distance between the two marks. Five measurements were taken for each direction of motion (elevation/depression and protraction/retraction). Five able-bodied men (ages 28-56) and five able-bodied women (ages 26-61) participated after giving informed consent according to the guidelines of the Northwestern University Institutional Review Board.

Force measurements were taken using the same 10 test subjects. Here the subject was seated comfortably and a padded load cell placed at the appropriate position and direction on or across from the acromion process. The subject was instructed to indicate when a comfortably maximal force was being applied against the load cell. At this point a reading was taken and recorded. Again, five measurements were taken corresponding to elevation, protraction, and retraction. (Shoulder depression could not be measured with this particular arrangement.)

\section{B. Goal 2}

In pointing tasks Fitts [9] defined the Index of Difficulty for a given pointing task as

$$
\mathrm{ID}=\log _{2}\left(\frac{\mathrm{D}}{\mathrm{d}}+1\right)
$$

where $\mathrm{D}$ is the distance between the starting and ending points for a particular target configuration, and $\mathrm{d}$ is the diameter of a circle enclosing some acceptable region around the ending point. The method consists of measuring the time required to move a pointing device from a starting point to targets of varying size and distance (and thus difficulty). Plots of time required (t) vs. ID exhibit a linear relationship of the form

$$
\mathrm{t}=\mathrm{m} \mathrm{ID}, \quad(2)
$$

where $m$ is the slope of a straight line passing through the origin. Pointing devices with low values of $\mathrm{m}$ are judged to perform better than those with high values. For this study a single experiment consisted of 16 conditions in which 11 small circles of diameter $\mathrm{d}$ are arranged around a larger circle of diameter D (Fig. 3). The test subject's task is to move a cursor on a display screen from circle 1 to circle 2 , to circle 3 , and so on until returning to circle 1 from circle 11. $\mathrm{d}$ and $\mathrm{D}$ change for each condition. Subjects moved the cursor via shoulder movement using each candidate control assembly/scheme.

\section{RESULTS AND DISCUSSION}

Overall performance in the displacement and force evaluations (Table 2) indicated that men and women exhibited similar movement range $(\sim 2.0-4.5 \mathrm{~cm})$ and force sensitivity $(\sim 24$ $\mathrm{N})$. Five of the ten subjects then participated in the controller evaluations. A linear mixedeffects model was used to assess the effect of the different assemblies and schemes on the time to complete the pointing over different indices of difficulties. The model included fixed 
effect terms for assembly, scheme, index of difficulty, assembly-by-time, scheme-by-time, and assembly-by-scheme-by-index of difficulty and the random effect terms on the intercept and the slope at subject, assembly, and scheme levels. There was no significant difference in performance between the three steel assemblies ( $\mathrm{p}=0.53$ ); but all of the steel assemblies performed significantly better than the potentiometer assembly $(\mathrm{p}=0.005)$. Furthermore, for all of the assemblies, the velocity control scheme was significantly better than the position control scheme $(\mathrm{p}=0.001)$.

We believe that the resistance to motion offered by the steel assemblies is responsible for their superior performance compared to that of the potentiometer. The steel assemblies constitute a limited implementation of extended physiological proprioception (EPP), as proposed by Simpson [11] and advanced by Doubler and colleagues [12, 13]. According to this concept, if the manner in which a mechanical device is controlled can be such that the operator is able to accurately perceive its static and dynamic characteristics through naturally arising proprioceptive sensations, the device becomes an artificial extension of the operator. By introducing a resistance to shoulder motion we provide the user a "feel" for the task which he/she can use to improve performance. Though the superiority of the velocity control scheme in this study contrasts with Doubler et al's conclusions [12], this was confirmed (for this control apparatus, at least) by the subjective evaluations given by participants after completing the pointing tasks. Though one could move the cursor from the starting position to the vicinity of the target circle more quickly with the position control scheme, locating and holding the cursor within the target proved more difficult and time consuming, as this in turn required precisely locating and holding the acromion process at a precise point in three-dimensional space. It thus proved easier overall to "push" the cursor towards the target and make mid-course corrections along the way, guiding it to the final position at the center of the target circle than to place it there using absolute shoulder position.

\section{CONCLUSIONS}

Design specifications for a 2 DOF prosthetic shoulder controller were developed, along with a tool to evaluate and compare different designs. Several candidate controllers were then evaluated. Designs based on velocity control and EPP performed better than those that were not. Here the evaluation task was to move a cursor on a display screen. The next step will be to migrate the controller to a prosthetic arm where it and the evaluation protocol and be tested and validated by actual subjects in their intended application. A particularly important aspect of this evaluation will be to compare the evaluation protocol's results with the more subjective assessments of the subjects. In order to realize the full benefits of EPP, the controller characteristics should match the dynamic characteristics of the controlled prosthetic limb. More generally, the controller is capable of controlling any two degrees of freedom, while the evaluation protocol could be applied to assess lower limb control and performance as well.

\section{Acknowledgments}

The author wishes to thank Dr. Mary Rodgers for her support and guidance based on a review of this article. This study was carried out at the Rehabilitation Institute of Chicago. 


\section{References}

1. Bayer DM, Lord RH, Swanker JW, Mortimer JT. A Two-Axis Shoulder Position Transducer for Control of Orthotic/Prosthetic Devices. IEEE Transactions on Industrial Electronics and Control Instrumentation. 1972; 19:61-64.

2. Mortimer, JT.; Bayer, DM.; Lord, RH.; Swanker, JW. Shoulder Position Transducer for Proportional Two Axis Control of Orthotic/Prosthetic Systems. In: Herberts, Kadefors Magnusson; Peterson, editors. The Control of Upper Extremity Prostheses and Orthoses. Charles C Thomas; Springfield, IL: 1974. p. 131-145.

3. Inman VT, Saunders JB, Abbot LC. Observations of the Function of the Shoulder Joint. Journal of Bone Joint Surgery. 1944; 26A:1-30.

4. Inman VT, Saunders JB. Observations of the Function of the Clav icle. California Medicine. 1946; 65:158-166. [PubMed: 18731101]

5. Kinzel GL, Hall AS, Billberry BM. Measurement of the Total Motion between Two Body Segments —I: Analytical Development. Journal of Biomechanics. 1972; 5:95-103.

6. Kinzel GL, Hall AS, Billberry BM. Measurement of the Total Motion Between Two Body Segments-II: Description of Application. Journal of Biomechanics. 1972; 5:283-293. [PubMed: 4666533]

7. Engin AE, Peindl RD. On the Biomechanics of Human Shoulder Complex-I: Kinematics for Determination of the Shoulder Complex Sinus. Journal of Biomechanics. 1987; 20:119-134. [PubMed: 3571293]

8. Peindl RD, Engin AE. On the Biomechanics of Human Shoulder Complex-II: Passive Resistive Properties Beyond the Shoulder Complex Sinus. Journal of Biomechanics. 1987; 20:103-117. [PubMed: 3571292]

9. Fitts PM. The Information Capacity of the Human Motor System in Controlling the Amplitude of Movement. Journal of Experimental Psychology. 1954; 47:381-391. [PubMed: 13174710]

10. Soukoreff TW, MacKenzie IS. Towards a Standard for Pointing Device Evaluation, Perspectives on 27 Years of Fitts' Law Research in HCI. International Journal of Human-Computer Studies. 2004; 61:751-789.

11. Simpson, DC. The Control of Upper-Extremity Prostheses and Orthoses. Herbert, et al., editors. Springfield, IL: 1974. p. 146-150.

12. Doubler JA, Childress JA. An Analysis of Extended Physiological Proprioception as a Control Technique. Journal of Rehabilitation Research and Development. 1984; 21:5-18. [PubMed: 6527290]

13. Doubler JA, Childress JA. Design and Evaluation of a Prosthesis Control System Based on the Concept of Extended Physiological Proprioception. Journal of Rehabilitation Research and Development. 1984; 21:19-318. [PubMed: 6527287] 


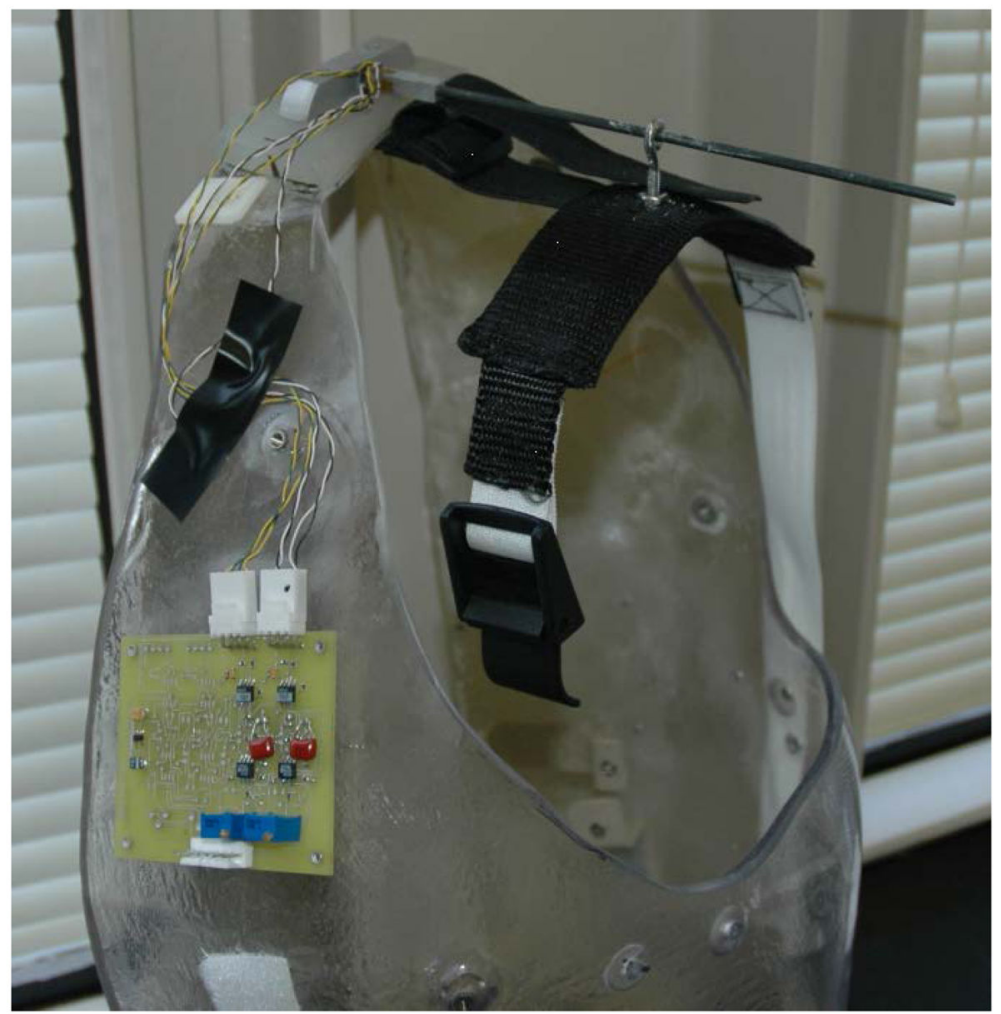

Figure 1.

Controller Configuration 


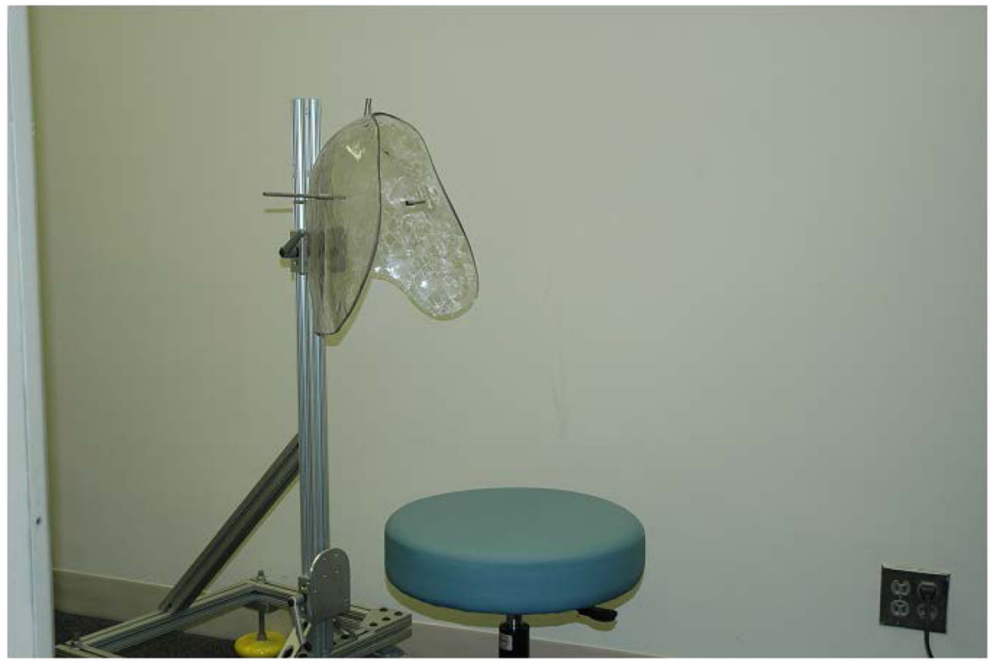

Figure 2.

Test Setup to Measure Shoulder Range of Motion and Force 


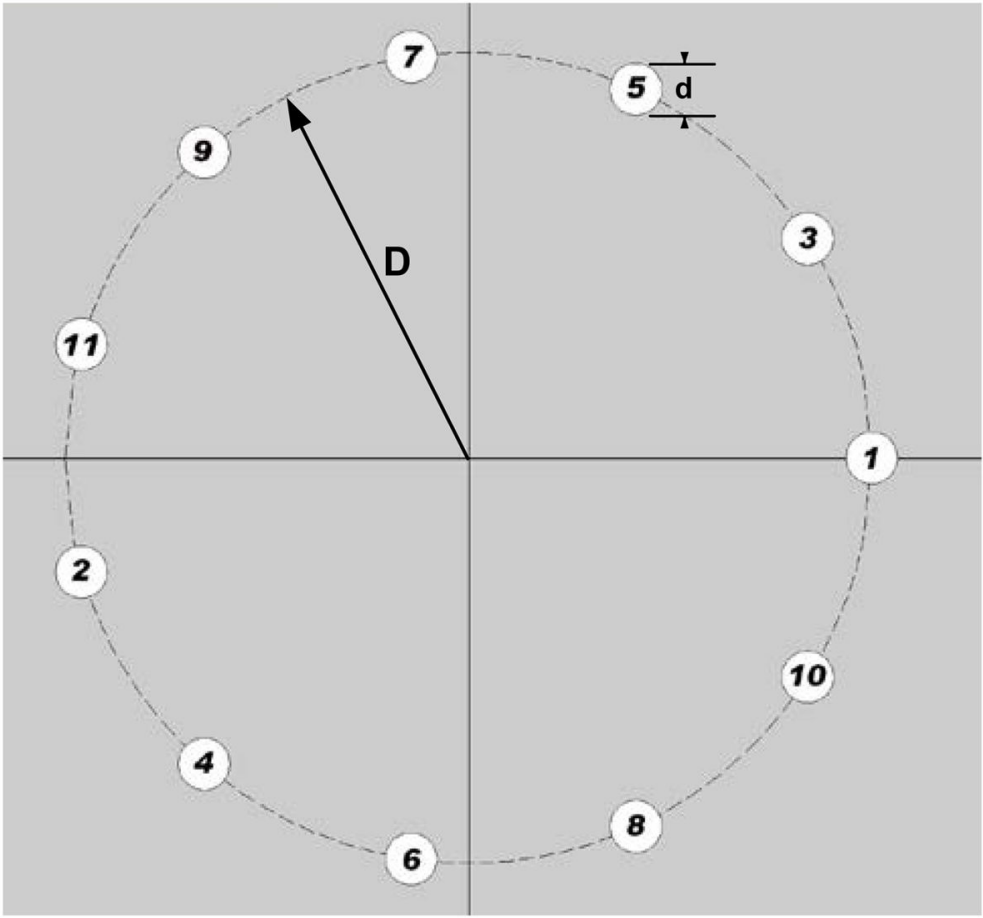

Figure 3.

Evaluative Task Based on Fitts Law 


\section{Table 1}

\section{Controller Characteristics}

\begin{tabular}{|c|c|}
\hline Rod Material & Stiffness (N/cm-deflection) \\
\hline $6.0 \mathrm{~mm}$ OD Steel & 4.1 \\
\hline $6.7 \mathrm{~mm}$ OD Steel & 6.6 \\
\hline $7.7 \mathrm{~mm}$ OD Steel & 11.1 \\
\hline 2 DOF Potentiometer & 0.0 \\
\hline
\end{tabular}

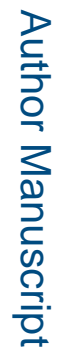

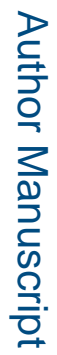

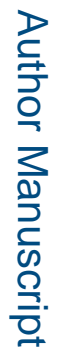

Conf Proc IEEE Eng Med Biol Soc. Author manuscript; available in PMC 2015 June 30. 
Table 2

Displacements and Forces Exerted at the Acromion Process

\begin{tabular}{|l|c|c|c|c|}
\hline Displacement $(\mathbf{c m})$ & Men & Women & Overall Average & $\sigma$ \\
\hline Elevation & 3.9 & 4.7 & 4.3 & 1.2 \\
\hline Protraction & 4.0 & 3.2 & 3.6 & 1.3 \\
\hline Retraction & 3.0 & 2.5 & 2.7 & 1.0 \\
\hline Depression & 1.8 & 1.9 & 1.9 & 1.1 \\
\hline Force (N) & & & & \\
\hline Elevation & 23.5 & 27.6 & 25.5 & 7.0 \\
\hline Protraction & 21.4 & 21.1 & 21.3 & 5.6 \\
\hline Retraction & 23.2 & 27.9 & 25.5 & 6.3 \\
\hline
\end{tabular}

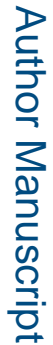

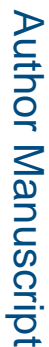

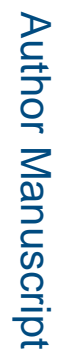

